\title{
Bickerstaff's encephalitis and the Miller Fisher syndrome
}

\author{
J B Winer
}

In 1951 Bickerstaff and Cloake described three cases of ophthalmoplegia under the title of "mesencephalitis and rhomboencephalitis" and suggested that the clinical features were due to a midbrain disturbance. ${ }^{1}$ In this original report the authors drew attention to the similarities of this condition to Guillain-Barré syndrome, although they concluded that a viral aetiology was more likely. Some 5 years later Miller Fisher published his famous paper on a syndrome of ophthalmoplegia, ataxia, and areflexia $^{2}$ and suggested that this syndrome was an unusual variant of Guillain-Barré syndrome because of absent reflexes and a raised protein in CSF. He proposed that the high CSF protein and the fact that ophthalmoplegia occurs in more classic Guillain-Barré syndrome suggested that the pathology would prove to be similar. Although the signs were difficult to explain on a purely peripheral basis he "reluctantly" concluded that they should be "interpreted as manifestations of an unusual and unique disturbance of peripheral neurons". The following year Bickerstaff published "further observations on a grave syndrome with benign prognosis" 3 which he continued to regard as a form of encephalitis. In this paper he reviewed his previous cases and described four others. Many of these patients had CSF pleocytosis and marked drowsiness. In one patient postmortem examination disclosed ballooning of myelin sheaths within the brain stem and diffuse loss of Purkinje cells in the cerebellum. Perivascular aggregations of lymphocytes were also seen in the frontal lobes. These changes were thought to suggest that much of the pathophysiology was due to cerebral oedema secondary to either a viral infection or resulting from hypersensitivity to an infection.

A subsequent publication from a group including Bickerstaff came down strongly on the side of an encephalitis in a paper ${ }^{4}$ that described 18 patients with the syndrome of which many had atypical features such as fever, extensor plantar signs, CSF pleocytosis, and coma. The authors reported low density changes in the brain stem on CT of a woman of 69 years. She subsequently died from the disease and at post mortem her midbrain showed foci of microglia, astrocytic proliferation, and cuffing of vessels.

Debate about the peripheral or central nature of these syndromes continued to rage in the literature. Cases with abnormal $\mathrm{CT}^{5}$ and more recently $M R$ imaging within the brain stem $^{6}$ have been reported. Even the absence of tendon reflexes has been ascribed to interruption of the tegmentum midbrain spinal cord pathways resulting in altered $\alpha$-motor neuron function. ${ }^{7}$ Observations on a patient with Miller Fisher syndrome who developed a supranuclear gaze palsy were cited as strong evidence of a central cause for the syndrome. ${ }^{8}$ In an editorial in the same volume of the Archives of Neurology Ropper ${ }^{9}$ hotly debated that conclusion, pointing out that some physical signs normally considered to indicate CNS involvement could be mimicked by peripheral nerve disease-for example, preservation of Bell's phenomenon, and supranuclear gaze palsy. Ropper proposed a mechanism for the ataxia in Miller Fisher syndrome ${ }^{10}$ based on a case that seemed to have a disparity of the proprioceptive information from muscle spindles and kinaesthetic information from joints and other proprioceptors.

A clearer understanding of the pathogenesis of Miller Fisher syndrome, Guillain-Barré syndrome, and ophthalmoplegia in brain stem encephalitis was clearly needed to resolve this debate about central versus peripheral involvement and the role of infective agents.

Studies over the past decade have thrown much light on the possible mechanism of immune mediated nerve damage. The finding that serum from the vast majority of patients with Miller Fisher syndrome ${ }^{1112}$ contains an antibody that reacts with the peripheral nerve ganglioside GQ1b provided a plausible explanation for at least part of the pathophysiology of the disease. Unlike the relation of antiganglioside antibodies with $\mathrm{GBS}^{13}$ the association of anti-GQ1b was so tight that it seemed very likely that these antibodies must in some way be pathogenetic. In 1994 Roberts et al showed that a serum factor caused neuromuscular blockade in patients with Miller Fisher syndrome by first increasing then blocking the firing of miniature end plate potentials in the mouse hemidiaphragm preparation. ${ }^{14}$ Studies by Buchwald et $a l^{15}$ have demonstrated neuromuscular block using anti-GQ1b IgG, strongly suggesting that this is the mediator of the symptoms in Miller Fisher syndrome. AntiGQ1b monoclonal antibody has also been shown to block neuromuscular transmission with an effect closely resembling the paralytic 
neurotoxin $\alpha$-lathratoxin in the mouse neuromuscular junction. ${ }^{16}$

Anti-GQ1b antibodies also cross react with lipolysaccharide in the bacterial coat of Campylobacter jejuni ${ }^{17}{ }^{18}$ providing a possible mechanism by which these antibodies might be formed in patients with Miller Fisher syndrome and indeed other forms of GuillainBarré syndrome. Cross reactivity with other organisms could explain cases of Miller Fisher syndrome not related to $C$ jejuni.

Further evidence of an important role for anti-GQ1b antibodies in Miller Fisher syndrome is the finding that the third, fourth, and sixth cranial nerves contain appreciable amounts of GQ1b ganglioside ${ }^{19}$ and can be stained using fluorescent labelled mouse antiGQ1b monoclonal. ${ }^{16}$ The frequency of involvement of ophthalmoparesis in myasthenia which itself results from neuromuscular blockade adds weight to this proposed pathogenetic mechanism. Despite the plausibility of this argument patients with Miller Fisher syndrome lack any clinical evidence of fatigability; nor is there any neurophysiological evidence to substantiate a defect in the neuromuscular junction.

Anecdotal reports of the response of patients with Miller Fisher syndrome to plasma exchange ${ }^{20}$ would be consistent with a pathogenic role for anti-GQ1b antibodies. The ultimate proof that anti-GQ1b antibodies mediate Miller Fisher syndrome requires the demonstration that passive transfer of antibody can mimic disease in vitro in an animal model but this has not yet been achieved.

How does Bickerstaff's brain stem encephalitis fit into our modern ideas about the pathogenesis of Miller Fisher syndrome? Patients with Guillain-Barré syndrome, Bickerstaff's encephalitis, and ophthalmoparesis without ataxia have also been shown to have anti-GQ1b antibodies to varying degrees. Yuki et $a l^{21}$ reported on a patient with drowsiness, ophthalmoplegia, abnormal EEG, and CSF pleocytosis that seemed to have overlapping GuillainBarré syndrome and Bickerstaff's encephalitis. Her serum IgG anti-GQ1b antibody titre was 1/6400 without antibodies to GM1, GD1b, or GT1b. Pathological findings included loss of large myelinated fires in the nerve roots, including the cranial nerves but normal midbrain pons and medulla. The cerebellum was moderately depleted of Purkinje cells and there were necrotic foci in the cerebral cortices. The authors proposed that Bickerstaff's encephalitis and Miller Fisher syndrome represent a single autoimmune disease with a wide range of symptoms including ophthalmoplegia and ataxia.

The association of anti-GQ1b antibodies with Bickerstaff's encephalitis is nowhere near so tight as with Miller Fisher syndrome but it does suggest a common pathogenesis for both diseases. A paper by Odaka and Herata ${ }^{22}$ has addressed this issue by collecting together 194 patients known to have anti-GQ1b antibodies and examining the clinical features of these cases and assigning to different diagnostic categories on the basis of their clinical, neurophysiological, and pathological features. They found many shared clinical and immunological findings within these groups including different frequencies of anti-GM1 and GD1a antibodies, the last occurring in $50 \%$ of cases of Guillain-Barré syndrome and only in $8 \%$ of Bickerstaff's encephalitis.

They propose the concept of an "anti-GQ1b antibody syndrome" which includes all these diseases despite different clinical features. Plasma exchange or IVIg might logically be justified in patients with anti-gQ1b antibodies and ophthalmoplegia whatever the primary clinical diagnosis. The evidence of benefit from these treatments in Bickerstaff's encephalitis however, remains anecdotal. ${ }^{23}$

It is amusing to conclude that despite the debate over the years about whether Miller Fisher syndrome was an immune mediated polyneuritis or a brain stem viral infection, both camps may well turn out to be able to claim credit. Perhaps anti-GQ1b antibodies are the key factor in mediating the ophthalmoplegia and these can be produced in various different ways in diseases that may affect both the peripheral nerves and less commonly the brain stem itself? Defects in neuromuscular transmission may be only one mechanism by which these antibodies mediate disease. Unravelling other mechanisms promises further fascinating insights into the cause of autoimmune neurological disease.

1 Bickerstaff ER, Cloake PCP. Mesenchephalitis and rhombencephalitis. BMF 1951;ii:77-81.

2 Fisher C. An unusal variant of acute idiopathic polyneuritis (syndrome of ophthalmoplegia, ataxia and areflexia). N Engl F Med 1956;225:57-75.

3 Bickerstaff E. Brain stem encephalitis: further observations on a grave syndrome with benign prognosis. BMF 1957;ii: 1384-7.

4 Al-Din AN AM, Bickerstaff ER, Harvey I. Brain stem encephalitis and the syndrome of Miller Fisher. A clinical encephalitis and the syndrome
study. Brain 1982;105:481-95.

5 Derakhshan I LJ, Kaufman B. Ophthalmoplegia, ataxia and hyporeflexia (Fisher's syndrome) with a midbrain lesion demonstrated by CT scanning. Eur Neurol 1979;18:361-6. 6 Landau WM, Glenn C, Dust G. MRI in Miller Fisher variant of Guillain-Barré syndrome. Neurology 1987;37:1431.

7 Barontini F SD. The nosological position of Fisher's syndrome (ophthalmoplegia, ataxia and areflexia). $\mathcal{F}$ Neurol 1983;229:33-44

8 Meienberg O RE. Supranuclear eye movement disorder in Fisher's syndrome of ophthalmoplegia, ataxia and areflexia. Arch Neurol 1983;40:402-5.

9 Ropper AH. The CNS in Guillain-Barré syndrome. Arch Neurol 1983;40:397-8.

10 Ropper AH, Shahani B. Proposed mechanism of ataxia in Fisher's syndrome. Arch Neurol 1983;40:537-8.

11 Chiba A, Kusunoki S, Shimizu T, et al. Serum IgG antibody to ganglioside GQ1b is a possible marker of Miller Fisher syndrome. Ann Neurol 1992;31:677-9.

12 Willison HJ, Veitch J, Paterson G, et al. Miller Fisher syndrome is associated with serum antibodies to GQ1b syndrome is associated with serum antibodies to GQ1b
ganglioside. 7 Neurol Neurosurg Psychiatry 1993;56:204-6.

13 Enders U, Karch H, Toyka KV, et al. The spectrum of immune responses to Campylobacter jejuni and glycoconjugates in Guillain-Barré syndrome and in other neuroimmunological disorders. Ann Neurol 1993;34:136-44.

14 Roberts $M$, Willison $\mathrm{H}$, Vincent $\mathrm{A}$, et al. Serum factor in Miller-Fisher variant of Guillain-Barré syndrome and neurotransmitter release . Lancet 1994;343:454-5.

15 Buchwald B, Dudel J, Toyka KV. Neuromuscular blockade by immunoglobulin $\mathrm{G}$ from patients with Miller Fisher syndrome. Ann N Y Acad Sci 1998;841:659-69.

16 Plomp JJ, Molenaar PC, O'Hanlon GM, et al. Miller Fisher anti-GQ1b antibodies: $\alpha$-latrotoxin-like effects on moto end plates. Ann Neurol 1999;45:189-99.

17 Yuki N. Molecular mimicry between gangliosides and lipopolysaccharides of Campylobacter jejuni isolated from patients with Guillain-Barré syndrome and Miller Fisher patients with Guillain-Barre syndrome and

18 Jacobs BC, Hazenberg MP, van Doorn PA, et al. Cross-reactive antibodies against gangliosides and Campylobacter jejuni lipopolysaccharides in patients with 
Guillain-Barré or Miller Fisher syndrome. 7 Infect Dis 1997; 175:729-33

19 Chiba A, Kusunoki S, Obata H, et al. Serum anti-GQ1b IgG antibody is associated with ophthalmoplegia in Miller Fisher syndrome and Guillain-Barré syndrome: clinical and immunohistochemical studies. Neurology 1993;43:1911-7.

20 Anonymous. Successful plasmapheresis in the Miller-Fisher syndrome. British Medical fournal Clinical Research Edition 1981;282:2055-6.
21 Yuki N, Wakabayashi K, Yamada $M$, et al. Overlap of Guillain-Barré syndrome and Bickerstaff's brainstem enGuillain-Barré syndrome and Bickersta

22 Odaka M Yuke N, Hirata K. Anti-GQ1b antibody syndrome: clinical and immunological spectrum. I Neurol Neurosurg Psychiatry 2001;70:50-5.

23 Ozawa $\mathrm{T}$, Onodera O, Inuzuka $\mathrm{T}$, et al. Efficacy of early plasmapheresis in Bickerstaff's encephalitis. Inter Med 1998;37:986-9.

\section{7th European Forum on Quality Improvement in Health Care}

\section{1-23 March 2002 Edinburgh, Scotland}

We are delighted to announce this forthcoming conference in Edinburgh. Authors are invited to submit papers (call for papers closes on Friday 5 October 2001) and delegate enquiries are welcome.

The themes of the Forum are:

- Leadership, culture change, and change management

- Achieving radical improvement by redesigning care

- Health policy for lasting improvement in health care systems

- Patient safety

- Measurement for improvement, learning, and accountability

- Partnership with patients

- Professional quality: the foundation for improvement

- Continuous improvement in education and training

- People and improvement.

Presented to you by the BMJ Publishing Group (London, UK) and Institute for Healthcare Improvement (Boston, USA). For more information contact: quality@bma.org.uk or look at the website www.quality.bmjpg.com. Tel: +44 (0)20 7383 6409; fax: +44 (0)20 73736869 . 